\title{
Diagnose Anthropologique A Partir Des Caractéristiques Dento-Squelettiques Et D'épaisseurs Des Tissus Mous Du Profil Facial: Etude Comparative Entre Population Autochtone Et Diaspora Etrangère
}

\author{
Assi Ar \\ Beugre. Jb \\ Département de Paléoanthropologie - Institut des Sciences \\ Anthropologiques de Développement d'Abidjan- UFHB Abidjan Côte \\ d'Ivoire \\ Laboratoire de Biomorphologie et d'Anthropologie Physique - UFR \\ Odonto-Stomatologie UFHB Abidjan Côte d'Ivoire
}

\section{Gnadoh Jj}

Département de Paléoanthropologie - Institut des Sciences Anthropologiques de Développement d'Abidjan- UFHB Abidjan Côte d'Ivoire

\section{Beugre-Kouassi Al}

Laboratoire de Biomorphologie et d'Anthropologie Physique - UFR Odonto-

Stomatologie UFHB Abidjan Côte d'Ivoire

doi: 10.19044/esj.2016.v12n36p286 URL:http://dx.doi.org/10.19044/esj.2016.v12n36p286

\begin{abstract}
Objective : The aim of this study was to search for the particularities in the dento-skeletal and soft tissue thickness of the facial cutaneous profile of Black Ivorians and White Lebanese.

Material and methods: This cross-sectional and descriptive study, concerned with numerical radiographs extracted from medical files of 119 adolescent patients [62 Ivorians (34 females and 28 males) and 57 Lebanese ( 31 females and 26 males)], from 11 to 16 years old, with skeletal class I and normodivergent faces, in first orthodontic surgery. From a drawing on acetate paper (Kodatrace type) and the materialization of true vertical and true horizontal (TVL and THL), 30 landmarks (19 cephalometric and 11 facial cutaneous profile landmarks) allowed the construction of lines and plans as well as 17 dento-skeletal measurements (10 angular et 7 linear) and 12 soft tissue thickness measurements. The data reliability was verified by Dahlberg's error method, while the $\mathrm{t}$ of student on independent series (signification threshold at 0,05) allowed to compare dento-skeletal characteristics and soft tissue thickness of the two groups.
\end{abstract}


The Results : Compared to White Lebanese, Black Ivorians present a facial prognathism resulting in more pronounced protrusion of alveolar bases $\left(\mathrm{SNA}=85,714^{\circ}, \mathrm{SNB}=81,321^{\circ}, \mathrm{A}-\mathrm{NPerp}=5,768^{\circ}\right)$ and vestibuloversion of central incisives $\left(\mathrm{I} / \mathrm{i}=111,679^{\circ}, \mathrm{I} / \mathrm{NA}=7,214 \mathrm{~mm}, \mathrm{i} / \mathrm{NB}=9,036 \mathrm{~mm}, \mathrm{NA}\right.$ and $\mathrm{NB}\left(\mathrm{I} / \mathrm{NA}=27,952^{\circ}\right.$ and $\left.\mathrm{i} / \mathrm{NB}=36,266^{\circ}\right)$ responsible of the obtuser facial convexity (NA-Pog $=169,338^{\circ}$ ). Indeed, this facial convexity reveals a very low prominence of the chin $(\mathrm{Pog}-\mathrm{NB}=-0,565 \mathrm{~mm})$. Also, the maxillomandibular discrepancy observed $\left(\mathrm{FMA}=24,516^{\circ}\right.$, greater), implies a more important facial growth kinetic in vertical direction with black Ivoirians. This is considerably reduced with females, with a less pronounced FMA angle compared to Lebanese females $\left[\mathrm{FMA}^{\circ}(\mathrm{P}=0,211 \mathrm{~ns})\right]$. The cutaneous effect of that dento-skeletal prognathism, is tangible through more protrusive lips hence, thicker - lips (Id-Ls=13,677 $\mathrm{mm}$ and id-Li=15,919 $\mathrm{mm}$ ). In this way, the observed substantial thickness is justified, of supporting peri-buccal soft tissue [lower lip's base (B-Sm=13,742 $\mathrm{mm})$ and the most inferior part of the chin $\left.\left(\mathrm{Me}-\mathrm{Me}^{\prime}=7,984 \mathrm{~mm}\right)\right]$. The two males groups present, however, a soft tissue thickness relatively similar on all the chin surface [Pog-Pog' $(\mathrm{P}=0,393$ ns); Gn-Gn' ( $\mathrm{P}=0,251 \mathrm{~ns})$; Me-Me' ( $\mathrm{P}=0,245 \mathrm{~ns})]$. The Black Ivorians' nose, platyrrhines, low and diving, present a thicker back (Rhi-Rhi' $=3,741 \mathrm{~mm})$ and thin supporting peripheral soft tissue thickness (ENA-Prn $=22,468 \mathrm{~mm}$, Prn perp/Sn-N'=12,935 mm, A-Sn= 13,097 mm) in general. In addition to those specific characteristics of Black Ivorians, males have a thicker soft tissue on the root of the nose $\left(\mathrm{N}^{-\mathrm{N}^{\prime}}=6,769 \mathrm{~mm}\right)$. Among the two female groups, the characteristics of soft tissue thickness are much more contrasted. Thus, besides the traditional morphological features (nose more prominent so, thicker - with Lebanese females, and thicker lips with Ivorian females), the forehead $\left(\mathrm{G}^{-} \mathrm{G}^{\prime}=6,441 \mathrm{~mm}\right)$ and the chin $\left(\mathrm{Pog}-\mathrm{Pog}{ }^{\prime}=13,941 \mathrm{~mm}, \mathrm{Gn}-\right.$ $\mathrm{Gn}^{\prime}=10,471 \mathrm{~mm}$, Me-Me' $=8,618 \mathrm{~mm}$ ) of Ivorian females are thicker.

Conclusion : The characteristics of dento-skeletal and soft tissue thickness of Black Ivorians and White Lebanese, reflect the anthropological diagnosis of their original ethnical groups. Facial prognathism and acuteness of facial convexity are observed, as well as greater soft tissue thickness of the inferior facial third (especially in females) traditionally particularizing the negroAfricans. However, a morphological proximity of the two ethnic groups is noticeable among males, concerning the soft tissue thickness of the chin, but also in females as for facial divergence. This study, because of what the results highlightened, has a forensic anthropology interest, and reveals the importance of radiological cephalometry.

Keywords: Ethnical groups, cephalometry, anthropological diagnosis, Black Ivorians, White Lebanese. 


\section{Résumé}

Objectif: L'objectif de cette étude a été de rechercher les particularités dento-squelettiques et d'épaisseurs des tissus mous du profil facial cutané des populations mélano-ivoiriennes et leuco-libanaises.

Matériels et méthodes: Cette étude transversale et descriptive, a porté sur des radiographies numériques extraites des dossiers médicaux de 119 patients adolescents [62 ivoiriens (34 filles et 28 garçons) et 57 libanais (31 filles et 26 garçons)] de 11 à 16 ans, de classe I squelettique et normodivergents, en première consultation orthodontique. A partir des tracés sur papier acétate (de type Kodatrace) et la matérialisation des verticale et horizontale vraies (THL et TVL), 30 points de repères (19 points de repères céphalométriques et 11 points sur le profil facial cutané localisés) ont permis la construction de lignes et plans ainsi que la réalisation de 17 mesures dento-squelettiques (10 angulaires et 7 linéaires) et 12 mesures d'épaisseurs des tissus mous. La fiabilité des données recueillies a été vérifiée par la méthode de Dahlberg pour le calcul d'erreur, tandis que le $t$ de student sur séries indépendantes (avec un seuil de signification à $0,05 \%$ ) a permis de comparer les caractéristiques dento-squelettiques et d'épaisseurs des tissus mous des deux groupes de sujets.

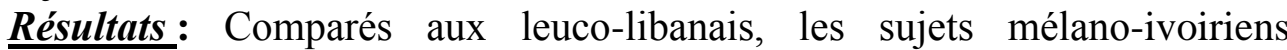
présentent un prognathisme facial se traduisant par une biprotrusion plus marquée des bases alvéolaires $\left(\mathrm{SNA}=85,714^{\circ}, \mathrm{SNB}=81,321^{\circ}\right.$, A-NPerp= $\left.5,768^{\circ}\right)$ et une vestibuloversion des incisives centrales $\left(\mathrm{I} / \mathrm{i}=111,679^{\circ}, \mathrm{I} / \mathrm{NA}=\right.$ $7,214 \mathrm{~mm}, \mathrm{i} / \mathrm{NB}=9,036 \mathrm{~mm}, \mathrm{NA}$ et $\mathrm{NB}\left(\mathrm{I} / \mathrm{NA}=27,952^{\circ}\right.$ et $\left.\mathrm{i} / \mathrm{NB}=36,266^{\circ}\right)$ à l'origine de l'angle de convexité faciale plus obtus (NA-Pog $=169,338^{\circ}$ ). Laquelle convexité faciale révèle en effet une très faible proéminence du menton (Pog-NB= $-0,565 \mathrm{~mm}$ ). Aussi, l'hyperdivergence faciale observée (angle $\mathrm{FMA}=24,516^{\circ}$, plus grand), traduit une cinétique de croissance faciale plus importante dans le sens vertical chez ces derniers. Celle-ci s'atténue considérablement chez les sujets féminins, avec un angle FMA moins marqué par rapport à celui des libanaises [FMA $\left.{ }^{\circ}(\mathrm{P}=0,211 \mathrm{~ns})\right]$. Le retentissement cutané de ce prognathisme dento-squelettique, est manifeste à travers des lèvres plus protruses, donc, plus épaisses (Id-Ls= 13,677 mm et id- $\mathrm{Li}=15,919$ $\mathrm{mm})$; justifiant ainsi les fortes épaisseurs des tissus mous péri-buccaux de soutien [base de la lèvre inférieure $(\mathrm{B}-\mathrm{Sm}=13,742 \mathrm{~mm})$ et partie la plus inférieure du menton $\left.\left(\mathrm{Me}-\mathrm{Me}^{\prime}=7,984 \mathrm{~mm}\right)\right]$ observées. Les deux groupes de sujets masculins présentent cependant, des épaisseurs de tissus mous relativement similaires sur toute la surface du menton [Pog-Pog' $(\mathrm{P}=0,393$ ns); Gn-Gn' ( $\mathrm{P}=0,251 \mathrm{~ns})$; Me-Me' ( $\mathrm{P}=0,245 \mathrm{~ns})]$. Le nez mélano-ivoirien, platyrhinien, bas et plongeant présente un dos plus épais (Rhi-Rhi' $=3,741$ $\mathrm{mm}$ ) et une faiblesse générale des épaisseurs de tissus mous périphériques de 
soutien (ENA-Prn $=22,468 \mathrm{~mm}, \operatorname{Prn}$ perp $/ \mathrm{Sn}-\mathrm{N}^{\prime}=12,935 \mathrm{~mm}, \mathrm{~A}-\mathrm{Sn}=13,097$ $\mathrm{mm}$ ). En plus de ces caractéristiques spécifiques aux sujets mélano-ivoiriens, les sujets masculins présentent des tissus mous plus épais à la racine du nez $\left(\mathrm{N}^{-\mathrm{N}^{\prime}}=6,769 \mathrm{~mm}\right)$. Chez les deux groupes de sujets féminins, les caractéristiques d'épaisseurs des tissus mous sont beaucoup plus tranchées. Ainsi, outre les traditionnels traits de caractères morphologiques (nez plus proéminent donc plus épais chez les libanaises et lèvres plus épaisses chez les ivoiriennes), le front $\left(\mathrm{G}_{-} \mathrm{G}^{\prime}=6,441 \mathrm{~mm}\right)$ et le menton $\left(\mathrm{Pog}-\mathrm{Pog}{ }^{\prime}=13,941 \mathrm{~mm}\right.$, $\mathrm{Gn}-\mathrm{Gn}{ }^{\prime}=10,471 \mathrm{~mm}$, Me-Me'=8,618mm) des filles ivoiriennes sont plus épais.

Conclusion: Les caractéristiques donto-squelettiques et d'épaisseurs des tissus mous des sujets mélano-ivoiriens et leuco-libanais, reflètent la diagnose anthropologique de leurs groupes ethniques d'origine. Le prognathisme facial, l'acuité de la convexité faciale ainsi que les fortes épaisseurs des tissus mous du tiers facial inférieur (surtout chez les sujets féminins) particularisant traditionnellement les sujets négro-africains, sont observés. Cependant une proximité morphologique des deux groupes ethniques s'observe chez les sujets masculins relativement aux épaisseurs des tissus mous du menton, mais également chez les sujets féminins quant à la divergence faciale. Cette étude, du fait de ce qu'elle a permis de révéler, présente un intérêt en anthropologie médico-légale et révèle l'importance de la céphalométrie radiologique.

Mots clés: Groupes ethniques, céphalométrie, diagnose anthropologique, mélano-ivoiriens, leuco-libanais.

\section{Introduction}

La connaissance des variations des épaisseurs des tissus mous faciaux, selon le morphotype squelettique, est importante aussi bien en anthropologie médico-légale que dans les disciplines orthodontiques. Dans la mesure où, la tête est la partie du corps qui présente les différences morphologiques les plus expressives pour caractériser les populations (Cherre, 2011) et que la physionomie faciale est fortement influencée par la forme et les dimensions du squelette sous-jacent (Fitz, 1981), divers travaux réalisés pour définir des normes céphalométriques sont exploités par l'anthropologie médico-légale.

Dans cette perspective, la littérature orthodontique expose un ensemble de données normatives particularisant des variétés ethniques, en relation avec l'âge et le sexe [enfants japonais (Utsuno et al. 2010), chinois (Gu et al. 2014), indiens (Meka et al. 2015) et Kikuyu du Kenya (Kapila, 1987), jeunes adultes émiratis (Abu-Tayyem et al. 2011), nigérians (Isiekwe et al. 2012, 2015), étudiants malaisiens (Purmal et al. 2013), adultes turques Celikoglu et al. (2015), libanais (Ayoub et al. 2008 ; El Hayeck et al. 2016), Sud-Africaines (Cavanagh et al. 2011)]. 
Ces normes dento-squelettiques et d'épaisseurs des tissus mous sont généralement comparées entre elles mais surtout avec des standards de références caucasiennes, contribuant ainsi à une caractérisation plus affinée de la diagnose anthropologique des populations.

A cet effet, dans leur étude portant sur deux populations de jeunes adultes (malaisiens indiens et malaisiens chinois), Purmal et al. (2013) notaient chez les malaisiens chinois, un maxillaire et une mandibule plus avancée, une hauteur de la face postérieure plus développée dans le sens vertical, une biprotrusion dentaire plus marquée s'accompagnant d'une plus forte protrusion des lèvres supérieure et inférieure. Les malaisiens indiens, en plus des caractéristiques contraires qui les qualifiaient, présentaient une hauteur de la face antérieure plus développée dans le sens vertical et un nez plus proéminent. Aussi, dans un registre quasi similaire, sur un groupe de sujets japonais de 6 à 18 ans en classe I squelettique, Utsuno et al. (2010) ont mis en relief une diagnose anthropologique traduisant des épaisseurs des tissus mous du profil cutané plus importantes au niveau de l'étage facial inférieur (par rapport aux normes caucasiennes et mélano-américaines).

Comparant les normes céphalométriques relatives aux tissus mous entre adultes turcs et américains caucasiens, Celebi et al. (2013) ont défini des caractéristiques turques exprimant une plus grande convexité faciale associée à une rétrusion mandibulaire, un angle face inférieure-gorge plus obtus, un angle naso-labial plus important et une plus forte protrusion de la lèvre supérieure, un sillon (sulcus) mento-labial plus profond, et un espace interlabial plus petit. Dans la même perspective, Freitas et al. (2010) sur deux groupes de nationaux adolescents brésiliens (caucasoïdes et mélanodermes) de classe I squelettique, faisaient observer que, les mélano-brésiliens se spécifiaient par un maxillaire et une mandibule plus protrus, une divergence antéropostérieure maxillo-mandibulaire, une plus grande convexité faciale, des incisives maxillaire et mandibulaire plus protruses entraînant une plus grande protrusion des lèvres supérieure et inférieure; tandis que les caucasoïdes se démarquaient par des caractéristiques de croissance verticale et une proéminence du menton plus accrues, ainsi qu'un angle naso-labial plus important. Ils notaient cependant une similarité morphologique au niveau des épaisseurs labiales entre ces deux groupes. La confrontation des spécificités ethniques aux standards caucasoïdes relativement à la morphotypologie squelettique et d'épaisseurs des tissus mous faciaux, a également été effectuée par Bagwan et al. (2015). Cette étude, portant sur un groupe d'adultes égyptiens a révélé que ces derniers se particularisaient par un profil facial plus convexe, un prognathisme maxillaire et une importante variabilité du prognathisme mandibulaire, une lèvre supérieure plus protruse, un angle naso-labial étroit, un espace interlabial plus grand, un sillon (sulcus) mento-labial plus profond, un angle face inférieure-gorge plus obtus 
$\mathrm{Au}$ regard de tous ces travaux, il est remarquable que les caractéristiques dento-squelettiques et d'épaisseurs des tissus mous des groupes de sujets étudiés conforte la variabilité morphologique des populations humaines, tout en renseignant sur les particularismes anthropologiques de différentiation. Il ne nous semble cependant pas avoir trouvé trace d'une étude portant sur la comparaison de sujets mélanodermes ivoiriens et diasporas étrangère, en l'occurrence leuco-libanaise. D'où l'objectif de cette étude qui a été de rechercher les particularités dentosquelettiques et d'épaisseurs des tissus mous du profil facial cutané des populations mélano-ivoiriennes et leuco-libanaises.

\section{Materiels et methodes}

La présente étude, de type transversal et descriptif, a porté sur des clichés de radiologie numérique issus des dossiers médicaux de 119 patients [62 ivoiriens (34 filles et 28 garçons) et 58 libanais (31 filles et 27 garçons) de 11 à 16 ans, en première consultation orthodontique. Ces radiographies standardisés et calibrées selon les normes de la céphalométrie clinique, ont toutes été produites par le service de radiologie de la PISAM (Polyclinique Sainte Anne-Marie) d'Abidjan, équipée des technologies les plus récentes en imagerie médicale. L'unité de radiologie numérique de cette structure était équipée d'un appareil de marque CRANEX Excel Ceph®, pourvu d'un céphalostat rotatif, donc permettant des acquisitions en incidence normalatéralis, norma-frontalis (dans le sens antéro-postérieur) et des radiographies panoramiques (dans le sens postéro-antérieur). Les images radiologiques étaient imprimées sur un film AGFA numérique ( 8 x 10 Inch) exposé à un courant électrique d'une puissance de $80 \mathrm{Kvp}$. La distance focale en usage dans ce centre était de $2 \mathrm{~m}$, tandis que la distance source-cassette était $50 \mathrm{~cm}$.

Ainsi, les sujets en posture cervicale neutre, les dents en intercuspidation maximale, les lèvres jointes, avaient la tête parfaitement orientée dans le plan horizontal de Frankfort et immobilisée dans le céphalostat en rotation frontale, perpendiculairement à la source d'émission du rayonnement $X$.

Sur un ensemble de 500 dossiers médicaux consultés, une sélection rigoureuse a été effectuée à un triple niveau. D'abord, les radiographies extraites ont été triées en fonction de leur exploitabilité, en retenant celles $(\mathrm{N}=350)$ qui présentaient un meilleur contraste d'image et dont les fiches de renseignements complémentaires indiquaient une bi-parentalité ivoirienne (pour les ivoiriens) et libanaise (pour les libanais). Ensuite, parmi ces clichés, ceux des patients dont l'âge était inclus entre 11 et 16 ans, en première consultation et n'ayant auparavant subi aucune intervention d'ordre orthodontique $(\mathrm{N}=250)$, ont été retenus. A la suite de ces premiers niveaux de sélections, un ensemble de tracés céphalométriques sur papier acétate à la 
lueur de l'éclairage du négatoscope, a permis de mesurer les angles ANB (position du maxillaire par rapport à la mandibule) et FMA (direction de croissance du squelette facial). 119 sujets [62 ivoiriens (34 filles et 28 garçons) et 57 libanais (31 filles et 26 garçons)] de classe I squelettique et normodivergentes ont été définitivement sélectionnés.

Les tracés réalisés sur les radiographies sélectionnées ont servi à une analyse céphalométrique plus globale, par la matérialisation de la verticale vraie $(\mathrm{TVL}=$ True verticale line) et l'horizontale vraie $(\mathrm{THL}=$ True horizontal line), la définition et la localisation de 19 points de repères sur le squelette facial et 11 points de repères sur le profil cutané facial.

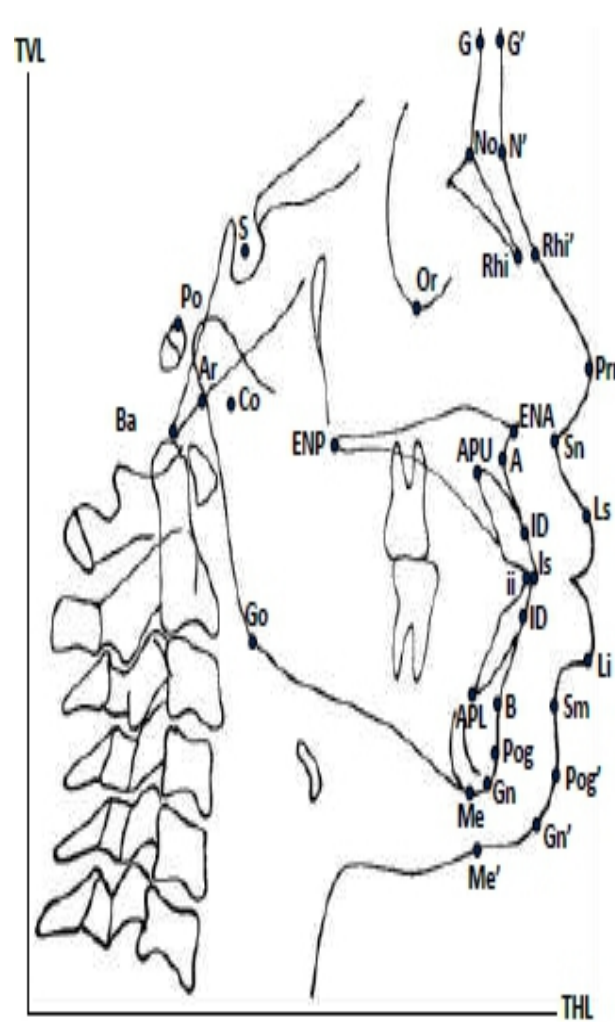

Figure 1 : Points de repères céphalométriques sur radiographie Norma-lateralis

\section{Points de repères squelette facial}

G: Glabelle osseux

No : Nasion osseux

Rhi: Rhinion osseux

S: Sella

Po : Porion

Ba : Basion

Ar : Articulare

Co : Condylion

Go: Gonion

Or: Orbitaire

ENA: Epine nasale antérieure

ENP : Epine nasale postérieure

A : Subspinal

APU : Apex incisive supérieure

Is : Incisive supérieure

Ii : Incisive inférieure

ID : Infradental

ID : Infradental

APL : Apex incisive inférieure

B : Supramental

Pog : Pogonion osseux

Gn : Gnathion osseux

THL : True horizontal line

TVL : True vertical line

A partir des points repères du squelette facial, un tracé de plans et de lignes ont permis la définition de 7 mesures linéaires et 10 mesures angulaires. Ces mesures réalisées étaient en rapport avec les différentes segments du squelette facial et certains de leurs rapports (composantes dento-alvéolaires, composantes maxillaires, composantes mandibulaires, composantes verticales, relations maxillo-mandibulaires). 


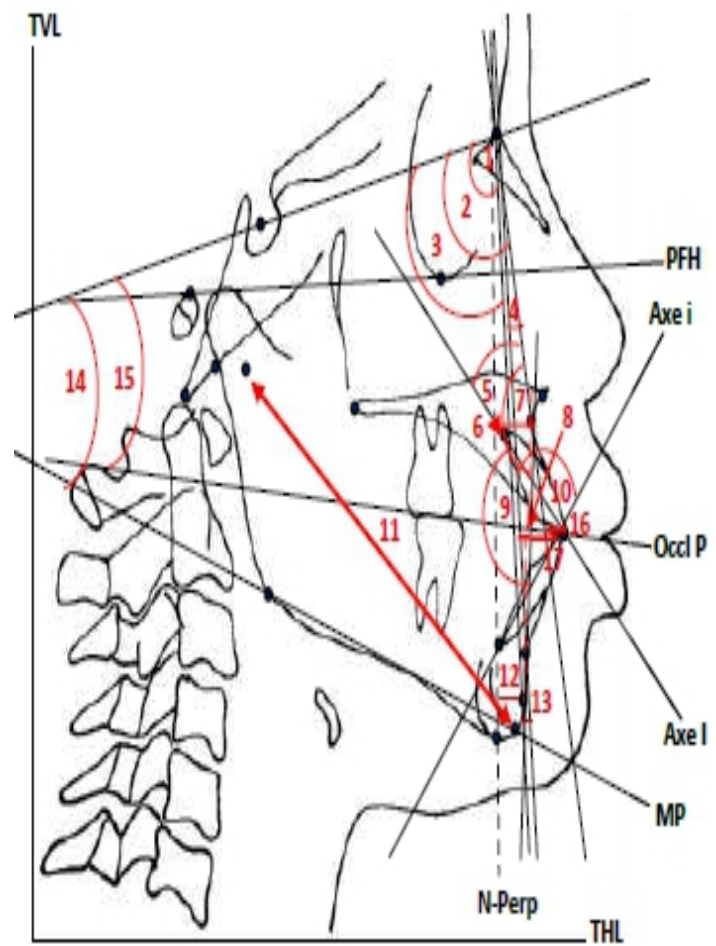

\section{Mesures Céphalométriques}

$1: \mathbf{S N B}^{\circ}=$ Angle formé par les lignes $\mathrm{SN}$ et $\mathrm{NB}$

2: $\mathbf{S N A}^{\circ}=$ Angle formé par les lignes $\mathrm{SN}$ et NA

$3: \mathbf{S N}-\mathbf{P o g}^{\circ}=$ Angle formé par les lignes $\mathrm{SN}$ et NPog

$4: \mathbf{A N B}^{\circ}=$ Angle formé par les lignes $\mathrm{AN}$ et NB

$5: \mathbf{I} / \mathbf{N A}^{\circ}=$ Angle formé par l'axe de l'incisive supérieure et la ligne NA

6 : A-NPerp $(\mathrm{mm})=$ Distance entre A et la perpendiculaire passant par $\mathrm{N}$

7: $\mathbf{N A}-\mathbf{P o g}=$ Angle de convexité faciale

8: $\mathbf{A o B o}=$ Distance entre les projections orthogonales de A et B sur le plan occlusal 9: $\mathbf{I} / \mathbf{i}^{\circ}=$ Angle inter-incisif

$10: \mathbf{i} / \mathbf{N A}{ }^{\circ}=$ Angle formé par l'axe de l'incisive supérieure et la ligne NB

11 : Co-Gn $(\mathrm{mm})=$ Longueur mandibulaire effective

12: Pog-NPerp $(\mathrm{mm})=$ Distance pogonion Perpendiculaire passant par nasion

13: Pog-NB $(\mathrm{mm})=$ Distance entre pogonion et la ligne NB

$14: \mathbf{F M A}^{\circ}=$ Angle formé par les plan de Frankfort et mandibulaire

$15: \mathbf{S N} / \mathbf{O c c l}^{\circ}=$ Angle formé par la ligne $\mathrm{SN}$ et le occlusal

$16: \mathbf{I} / \mathbf{N A}(\mathrm{mm})=$ Distance entre la pointe de l'incivive supérieure et la ligne NA

$17: \mathbf{i} / \mathbf{N B}(\mathrm{mm})=$ Distance entre la pointe de l'incisive supérieure et la ligne NA

Figure 2 : Points, lignes et mesures céphalométriques

Aussi, les épaisseurs des tissus mous ont-elles été évaluées à partir de la mesure des distances entre les points de repères du profil cutané, leurs homologues et certains points sur le squelette facial. Au nombre de 11, ces mesures d'épaisseurs des tissus sont matérialisées dans le schéma ci-dessous.

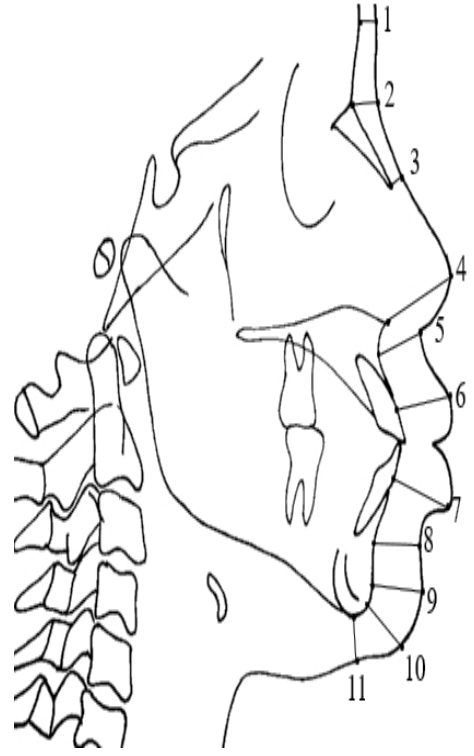

\section{Mesures d'épaisseurs des tissus mous}

1 - G-G' : Epaisseurs des tissus mous au niveau de la glabelle

2- N-N' : Epaisseurs des tissus mous au niveau du nasion

3- Rhi-Rhi' : Epaisseurs des tissus mous au niveau du rhinion

4- ENA-Prn : Proéminence du nez

5- A-Sn : Epaisseurs des tissus mous au niveau de la base du nez

6- ID-Ls : Epaisseurs de la lèvre supérieure

7- ID-Li : Epaisseurs de la lèvre inférieure

\section{8- B-Sm :}

9- Pog-Pog' : Epaisseurs des tissus mous au niveau du pogonion

10- Gn-Gn' : Epaisseurs des tissus mous au niveau du gnathion

11- Me-Me' : Epaisseurs des tissus mous au niveau du menton

Figure 3 : Mesures d'épaisseurs des tissus mous du profil facial cutané 
La fiabilité et la reproductibilité des données obtenues ont été testées à travers leur reprise, par le même opérateur, sur 20 radiographies sélectionnées au hasard (test du nombre au hasard par application de la table du nombre au hasard de Babington Smith et Kendall à la liste des sujets) 2 semaines plus tard. Le calcul d'erreur de mesure a ensuite été effectué, en utilisant la méthode d'erreur proposée par Dalberg (1940) où $\mathrm{Me}=\sqrt{\Sigma} \mathrm{d}^{2} / 2 \mathrm{n}$ ( $d$ est la différence entre les premières mesures et les deuxièmes mesures et $n$ le nombre de personnes tirées au hasard). Une analyse statistique descriptive a permis de déterminer les moyennes de valeurs relatives aux caractéristiques dento-squelettiques et d'épaisseurs des tissus mous du profil cutané facial au sein des deux groupes populationnels. Le $\mathrm{t}$ de student sur séries indépendantes (seuil signification 0,05\%) a été utilisé pour comparer les deux groupes de sujets féminins ; Tandis que la comparaison des deux populations masculines a été effectuée au moyen du test de Mann- Whithney sur séries indépendantes (seuil signification $0,05 \%$ ).

\section{Resultats}

Tableau I : Fiabilité et reproductibilité des mesures

\begin{tabular}{|c|c|c|}
\hline \multicolumn{2}{|c|}{ Variables } & M.E Dahlberg \\
\hline \multirow{20}{*}{ 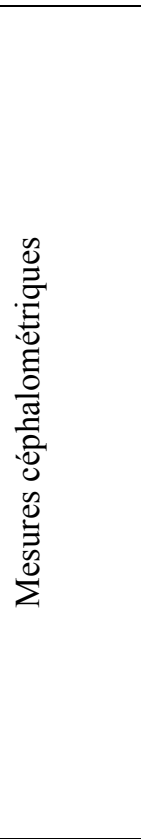 } & $\mathrm{I} / \mathrm{NA}^{\circ}$ & 0,060 \\
\hline & I/NA & 0,019 \\
\hline & $\mathrm{i} / \mathrm{NB}^{\circ}$ & 0,011 \\
\hline & $\mathrm{i} / \mathrm{NB}$ & 0,002 \\
\hline & $\mathrm{I} / \mathrm{i}^{\circ}$ & 0,039 \\
\hline & \multicolumn{2}{|c|}{ Composantes maxillaires } \\
\hline & $\mathrm{SNA}^{\circ}$ & 0,013 \\
\hline & A-N perp & 0,017 \\
\hline & \multicolumn{2}{|c|}{ Composantes mandibulaires } \\
\hline & $\mathrm{SNB}^{\circ}$ & 0,017 \\
\hline & Co-Gn & 0,019 \\
\hline & Pog-N perp & 0,045 \\
\hline & Pog-NB & 0,043 \\
\hline & $\mathrm{ANB}^{\circ}$ & 0,019 \\
\hline & AoBo & 0,037 \\
\hline & NA-Pog ${ }^{\circ}$ & 0,013 \\
\hline & \multicolumn{2}{|c|}{ Composantes verticales } \\
\hline & FMA $^{\circ}$ & 0,037 \\
\hline & $\mathrm{SN} \mathrm{GoGN}^{\circ}$ & 0,011 \\
\hline & $\mathrm{SN} / \mathrm{Occl}^{\circ}$ & 0,037 \\
\hline \multirow{7}{*}{ 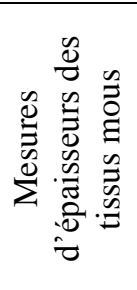 } & G-G' & 0,316 \\
\hline & N-N' & 1,423 \\
\hline & Rhi-Rhi' & 0,316 \\
\hline & Prn perp/SN-N' & 0,948 \\
\hline & ENA-Prn & 0,790 \\
\hline & A-Sn & 0,632 \\
\hline & Is-Ls & 0,948 \\
\hline
\end{tabular}




\begin{tabular}{|c|c|c|}
\hline \multirow{4}{*}{} & If-Li & 1,106 \\
\cline { 2 - 3 } & B-Sm & 0,790 \\
\cline { 2 - 3 } & Pog-Pog' & 0,632 \\
\cline { 2 - 3 } & Gn-Gn' & 0,948 \\
\cline { 2 - 3 } & Me-Me' & 0,632 \\
\hline
\end{tabular}

Les mesures réalisées dans cette étude présentent, dans l'ensemble, une cohérence et une précision acceptables.

Tableau II : Comparaison des caractéristiques dento-squelettiques et d'épaisseurs des tissus mous des sujets ivoiriens et libanais

\begin{tabular}{|c|c|c|c|c|c|c|}
\hline \multicolumn{2}{|c|}{ Variables } & \multicolumn{2}{|c|}{ Ivoiriens $\mathrm{N}=62$} & \multicolumn{2}{|c|}{ Libanais $\mathrm{N}=58$} & \multirow[t]{2}{*}{ P value } \\
\hline \multirow{33}{*}{ 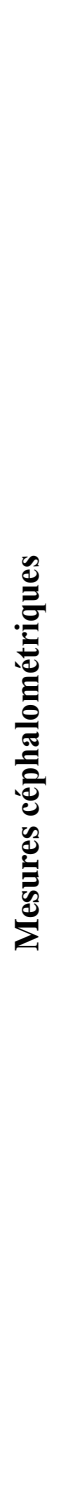 } & & Moyenne & $\begin{array}{l}\text { Ecart- } \\
\text { type }\end{array}$ & Moyenne & $\begin{array}{c}\text { Ecart- } \\
\text { type }\end{array}$ & \\
\hline & \multicolumn{6}{|c|}{ Composantes dento-alvéolaires } \\
\hline & $\mathrm{I} / \mathrm{NA}^{\circ}$ & 27,952 & 7,897 & 24,912 & 6,180 & $0,022 *$ \\
\hline & I/NA & 6,637 & 2,691 & 4,237 & 2,018 & $<$ \\
\hline & & & & & & $0,0001 *$ \\
\hline & $\mathrm{i} / \mathrm{NB}^{\circ}$ & 36,266 & 6,227 & 27,622 & 5,159 & $<$ \\
\hline & & & & & & $0,0001 *$ \\
\hline & $\mathrm{i} / \mathrm{NB}$ & 9,411 & 2,643 & 4,860 & 2,165 & $<$ \\
\hline & & & & & & $0,0001 *$ \\
\hline & $\mathrm{I} / \mathrm{i}^{\circ}$ & 109,927 & 13,658 & 124,667 & 8,609 & $<$ \\
\hline & \multicolumn{4}{|c|}{ Composantes maxillaires } & & $0,0001 *$ \\
\hline & SNA $^{\circ}$ & 85,742 & 3,951 & & 3,683 & $<$ \\
\hline & & & & & & $0,0001 *$ \\
\hline & A-N perp & 7,621 & 5,260 & 0,421 & 4,873 & $<$ \\
\hline & \multicolumn{6}{|c|}{ Composantes mandibulaires } \\
\hline & $\mathrm{SNB}^{\circ}$ & 80,750 & 4,433 & 77,403 & 3,688 & $<$ \\
\hline & Co-Gn & 99,879 & 10,643 & 96,421 & 10,474 & $0,0001^{*}$ \\
\hline & & & & & & $0,077 \mathrm{~ns}$ \\
\hline & Pog-N perp & 5,532 & 8,260 & 3,386 & 5,013 & \\
\hline & Pog-NB & $-0,565$ & 0,977 & 1,535 & 0,920 & $<$ \\
\hline & & & & & & $0,0001 *$ \\
\hline & \multicolumn{6}{|c|}{ Relations maxillomandibulaires } \\
\hline & $\mathrm{ANB}^{\circ}$ & 4,887 & 2,706 & 3,053 & 2,026 & $<$ \\
\hline & & & & & & $0,0001 *$ \\
\hline & AoBo & 2,113 & 3,930 & 2,632 & 2,193 & $0,382 \mathrm{~ns}$ \\
\hline & NA-Pog ${ }^{\circ}$ & 169,338 & 5,487 & 175,509 & 3,793 & $<$ \\
\hline & & & & & & $0,0001 *$ \\
\hline & \multicolumn{6}{|c|}{ Composantes verticales } \\
\hline & FMA $^{\circ}$ & 24,516 & 4,915 & 22,009 & 3,194 & $0,001 *$ \\
\hline & $\mathrm{SN} / \mathrm{GoGN}^{\circ}$ & 33,556 & 5,664 & 33,307 & 4,081 & 0,785 \\
\hline & & & & & & ns \\
\hline & $\mathrm{SN} / \mathrm{Occl}^{\circ}$ & 18,371 & 10,833 & 18,754 & 4,235 & 0,803 \\
\hline & & & & & & ns \\
\hline
\end{tabular}




\begin{tabular}{|c|c|c|c|c|c|c|}
\hline \multirow{22}{*}{ 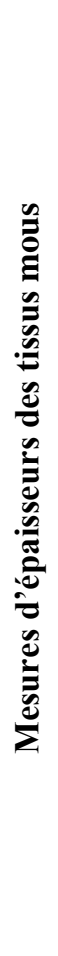 } & G-G' & & & & & 0,066 \\
\hline & & 6,161 & 1,321 & 5,741 & 1,148 & $\mathrm{~ns}$ \\
\hline & $\mathrm{N}-\mathrm{N}^{\prime}$ & & & & & 0,312 \\
\hline & & 6,258 & 1,305 & 6,500 & 1,301 & $\mathrm{~ns}$ \\
\hline & Rhi-Rhi' & & & & & $<$ \\
\hline & & 3,741 & 1,253 & 2,586 & 0,817 & $0,0001 *$ \\
\hline & ENA-Prn & & & & & $<$ \\
\hline & & 22,468 & 2,912 & 26,121 & 3,839 & $0,0001 *$ \\
\hline & Prn perp/Sn-N' & 12,935 & & & & $<$ \\
\hline & & & 1,871 & 14,690 & 2,349 & $0,0001 *$ \\
\hline & $\mathrm{A}-\mathrm{Sn}$ & 13,097 & & & & $<$ \\
\hline & & & 1,905 & 15,586 & 2,884 & $0,0001 *$ \\
\hline & ID-Ls & 13,677 & & & & $0,014 *$ \\
\hline & Id-Li & 15,919 & 2,448 & 12,500 & 2,735 & $<$ \\
\hline & & & 2,498 & 13,741 & 2,275 & $0,0001 *$ \\
\hline & B-Sm & 13,742 & & & & $<$ \\
\hline & & & 3,136 & 11,500 & 1,894 & $0,0001 *$ \\
\hline & Pog-Pog' & 13,306 & & & & $0,001 *$ \\
\hline & & & 3,145 & 11,500 & 2,703 & \\
\hline & Gn-Gn & 9,903 & 2,684 & 9,103 & 2,447 & $\begin{array}{l}0,091 \\
n s\end{array}$ \\
\hline & Me-Me' & 7,983 & & & & $0,037 *$ \\
\hline & & & 2,459 & 7,086 & 2,195 & \\
\hline
\end{tabular}

Comparés aux sujets leuco-libanais, les sujets ivoiriens présentent un prognathisme facial se traduisant par une biprotrusion plus marquée des bases alvéolaires $\left(\mathrm{SNA}=85,714^{\circ}, \mathrm{SNB}=81,321^{\circ}, \mathrm{A}-\mathrm{NPerp}=5,768^{\circ}\right)$ et une vestibuloversion des incisives centrales $\left(\mathrm{I} / \mathrm{i}=111,679^{\circ}, \mathrm{I} / \mathrm{NA}=7,214 \mathrm{~mm}\right.$, $\mathrm{i} / \mathrm{NB}=9,036 \mathrm{~mm}, \mathrm{NA}$ et $\mathrm{NB}\left(\mathrm{I} / \mathrm{NA}=27,952^{\circ}\right.$ et $\left.\mathrm{i} / \mathrm{NB}=36,266^{\circ}\right)$ à l'origine de l'angle de convexité faciale plus obtus $\left(\mathrm{NA}-\mathrm{Pog}=169,338^{\circ}\right)$. Laquelle convexité faciale révèle en effet une très faible proéminence du menton (Pog$\mathrm{NB}=-0,565 \mathrm{~mm}$ ). Aussi, l'hyperdivergence faciale observée (angle $\mathrm{FMA}=24,516^{\circ}$, plus grand), traduit une cynétique de croissance faciale plus importante dans le sens vertical chez ces derniers.

Le retentissement cutané de cette architecture dento-squelettique prognathe, est manifeste à travers des lèvres plus protruses, donc, plus épaisses (Id-Ls=13,677 $\mathrm{mm}$ et id-Li=15,919 $\mathrm{mm}$ ) ; justifiant ainsi les fortes épaisseurs des tissus mous péri-buccaux de soutien [base lèvre inférieure (B$\mathrm{Sm}=13,742 \mathrm{~mm})$ et au niveau du menton $\left.\left(\mathrm{Me}-\mathrm{Me}^{\prime}=7,984 \mathrm{~mm}\right)\right]$ observées.

Le nez des sujets mélano-ivoiriens, platyrhinien, bas et plongeant présente, comparativement aux sujets leuco-libanais, un dos plus épais (RhiRhi' $=3,741 \mathrm{~mm}$ ) et une faiblesse générale des épaisseurs des tissus mous péri-nasaux de soutien (ENA-Prn $=22,468 \mathrm{~mm}$, Prn perp/Sn-N'=12,935 mm, $\mathrm{A}-\mathrm{Sn}=13,097 \mathrm{~mm}$ ). 
Tableau III : Comparaison des caractéristiques dento-squelettiques et d'épaisseurs des tissus mous des garçons ivoiriens et libanais

\begin{tabular}{|c|c|c|c|c|c|c|}
\hline & \multirow[t]{2}{*}{ Variables } & \multicolumn{2}{|c|}{$\begin{array}{c}\text { Garçons ivoiriens } \\
\mathrm{N}=\mathbf{2 8}\end{array}$} & \multicolumn{2}{|c|}{$\begin{array}{l}\text { Garçons libanais } \\
\qquad N=26\end{array}$} & \multirow[t]{2}{*}{$P$ value } \\
\hline \multirow{23}{*}{ 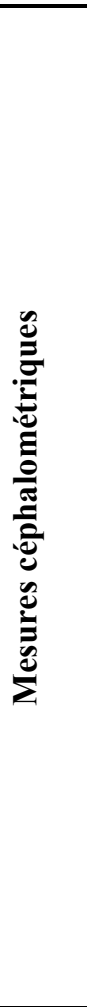 } & & Moyenne & $\begin{array}{l}\text { Ecart- } \\
\text { type }\end{array}$ & Moyenne & $\begin{array}{l}\text { Ecart- } \\
\text { type }\end{array}$ & \\
\hline & \multicolumn{6}{|c|}{ Composantes dento-alvéolaires } \\
\hline & $\mathrm{I} / \mathrm{NA}^{\circ}$ & 29,821 & 7,277 & 26,769 & 5,715 & $\begin{array}{l}0,071 \\
\mathrm{~ns}\end{array}$ \\
\hline & I/NA & 7,214 & 2,888 & 4,808 & 1,980 & $0,001 *$ \\
\hline & $\mathrm{i} / \mathrm{NB}^{\circ}$ & 34,089 & 5,657 & 28,654 & 5,756 & $0,002 *$ \\
\hline & $\mathrm{i} / \mathrm{NB}$ & 9,036 & 2,553 & 5,462 & 2,416 & $<0,0001 *$ \\
\hline & $\mathrm{I} / \mathrm{i}^{\circ}$ & 111,679 & 9,238 & 122,115 & 8,397 & $0,000 *$ \\
\hline & \multicolumn{6}{|c|}{ Composantes maxillaires } \\
\hline & SNA $^{\circ}$ & 85,714 & 3,398 & 81,230 & 3,892 & $0,000 *$ \\
\hline & A-N perp & 5,768 & 5,592 & $-3,576$ & 3,765 & $<0,0001 *$ \\
\hline & \multicolumn{6}{|c|}{ Composantes mandibulaires } \\
\hline & $\mathrm{SNB}^{\circ}$ & 81,321 & 3,984 & 77,807 & 3,889 & $0,004 *$ \\
\hline & Co-Gn & 99,161 & 11,688 & 99,346 & 9,291 & $0,842 \mathrm{~ns}$ \\
\hline & Pog-N perp & 3,375 & 9,722 & 3,019 & 5,045 & $0,917 \mathrm{~ns}$ \\
\hline & Pog-NB & $-0,411$ & 1,046 & 1,423 & 0,924 & $<0,0001 *$ \\
\hline & \multicolumn{6}{|c|}{ Relations maxillomandibulaires } \\
\hline & $\mathrm{ANB}^{\circ}$ & 4,161 & 2,520 & 3,269 & 2,355 & $0,040 *$ \\
\hline & AoBo & 2,589 & 4,344 & 2,423 & 2,352 & $0,951 \mathrm{~ns}$ \\
\hline & NA-Pog ${ }^{\circ}$ & 170,661 & 5,207 & 175,654 & 3,655 & $0,001 *$ \\
\hline & \multicolumn{6}{|c|}{ Composantes verticales } \\
\hline & FMA $^{\circ}$ & 24,821 & 4,234 & 20,962 & 3,412 & $0,001 *$ \\
\hline & $\mathrm{SN} / \mathrm{GoGN}^{\circ}$ & 32,321 & 5,221 & 31,962 & 4,547 & $0,722 \mathrm{~ns}$ \\
\hline & $\mathrm{SN} / \mathrm{Occl}^{\circ}$ & 18,982 & 15,129 & 18,769 & 4,339 & $0,294 \mathrm{~ns}$ \\
\hline \multirow{12}{*}{ 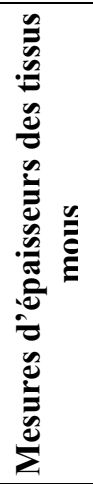 } & G-G' & 5,821 & 1,090 & 6,115 & 1,143 & $0,370 \mathrm{~ns}$ \\
\hline & N-N' & 6,071 & 1,120 & 6,769 & 1,032 & $0,010^{*}$ \\
\hline & Rhi-Rhi' & 3,714 & 0,854 & 2,885 & 0,816 & $0,001 *$ \\
\hline & ENA-Prn & 22,750 & 3,216 & 27,000 & 3,940 & $0,000 *$ \\
\hline & Prn perp/Sn-N' & 12,571 & 1,874 & 14,538 & 2,420 & $0,002 *$ \\
\hline & A-Sn & 13,357 & 2,004 & 15,962 & 2,764 & $0,001 *$ \\
\hline & ID-Ls & 13,857 & 2,649 & 12,885 & 2,566 & $0,101 \mathrm{~ns}$ \\
\hline & Id-Li & 16,357 & 2,896 & 14,731 & 1,909 & $0,011 *$ \\
\hline & B-Sm & 14,393 & 3,552 & 12,115 & 1,796 & $0,003 *$ \\
\hline & Pog-Pog' & 12,536 & 3,585 & 12,038 & 2,720 & $0,393 \mathrm{~ns}$ \\
\hline & Gn-Gn' & 9,214 & 2,529 & 9,923 & 1,958 & $0,251 \mathrm{~ns}$ \\
\hline & Me-Me' & 6,964 & 1,551 & 7,462 & 1,555 & $0,245 \mathrm{~ns}$ \\
\hline
\end{tabular}

*Valeur de P significative (P < 0,005) ns= Valeur de P non significative -Test student-

L'architecture dento-squelettique et les épaisseurs des tissus mous du profil cutané des sujets masculins ivoiriens et libanais, mettent en relief, la diagnose anthropologique reconnue à leurs groupes ethniques d'origine. Le prognathisme facial $\left(\mathrm{SNA}=85,714^{\circ}, \mathrm{SNB}=81,321^{\circ}, \mathrm{A}-\mathrm{NPerp}=5,768^{\circ}, \mathrm{I} / \mathrm{i}=\right.$ $111,679^{\circ}, \mathrm{I} / \mathrm{NA}=7,214 \mathrm{~mm}, \mathrm{i} / \mathrm{NB}=9,036 \mathrm{~mm}, \mathrm{NA}$ et $\mathrm{NB}\left(\mathrm{I} / \mathrm{NA}=27,952^{\circ}\right.$ et $\mathrm{i} / \mathrm{NB}=36,266^{\circ}, \quad \mathrm{NA}-\mathrm{Pog}=169,338^{\circ}$ ) et l'hyperdivergence faciale 
$\left(\mathrm{FMA}=24,516^{\circ}\right)$ ainsi que les épaisseurs du dos du nez (Rhi-Rhi'=3,714 $\mathrm{mm}$ ), des lèvres ( $\mathrm{Id}-\mathrm{Ls}=13,857 \mathrm{~mm}$ et id-Li $=16,357 \mathrm{~mm}$ ) et des tissus mous péri-bucaux $\left(\mathrm{B}-\mathrm{Sm}=14,393 \mathrm{~mm}, \mathrm{Me}-\mathrm{Me}{ }^{\prime}=7,984 \mathrm{~mm}\right)$ plus importantes, caractérisent les sujets masculins mélano-ivoiriens. Tandis que les sujets masculins leuco-libanais, sont caractérisés par un menton plus proéminent $\left(\mathrm{Pog}-\mathrm{NB}=1,423^{\circ}\right)$, un nez plus proéminent donc plus épais (ENA-Prn= $27,000 \mathrm{~mm})$, des épaisseurs de tissus mous plus importantes à la racine (N$\left.\mathrm{N}^{\prime}=6,769 \mathrm{~mm}\right)$ et à la limite inférieure du nez $(\mathrm{A}-\mathrm{Sn}=15,962 \mathrm{~mm})$.

Il est cependant notable que les épaisseurs des tissus mous, sont plus proches entre les deux groupes de sujets masculins, dans toute la région périphérique du menton [Pog-Pog' ( $\mathrm{P}=0,393 \mathrm{~ns})$; Gn-Gn' ( $\mathrm{P}=0,251 \mathrm{~ns})$; Me$\left.\mathrm{Me}^{\prime}(\mathrm{P}=0,245 \mathrm{~ns})\right]$.

Tableau IV : Comparaison des caractéristiques dento-squelettiques et d'épaisseurs des tissus mous des filles ivoiriennes et libanaises

\begin{tabular}{|c|c|c|c|c|c|c|}
\hline \multirow[t]{2}{*}{ 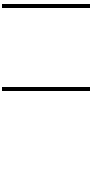 } & \multirow[t]{2}{*}{ Variables } & \multicolumn{2}{|c|}{$\begin{array}{l}\text { Filles ivoiriennes } \\
\qquad \mathbf{N}=\mathbf{3 4}\end{array}$} & \multicolumn{2}{|c|}{$\begin{array}{l}\text { Filles libanaises } \\
\qquad \mathbf{N}=\mathbf{3 1}\end{array}$} & \multirow[t]{2}{*}{ P value } \\
\hline & & Moyenne & $\begin{array}{l}\text { Ecart- } \\
\text { type }\end{array}$ & Moyenne & $\begin{array}{l}\text { Ecart- } \\
\text { type }\end{array}$ & \\
\hline \multirow{27}{*}{ 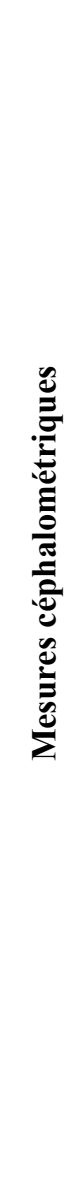 } & \multicolumn{6}{|c|}{ Composantes dento-alvéolaires } \\
\hline & $\mathrm{I} / \mathrm{NA}^{\circ}$ & 26,412 & 8,157 & 23,355 & 6,210 & $0,096 \mathrm{~ns}$ \\
\hline & I/NA & 6,162 & 2,458 & 3,758 & 1,953 & $<0,0001 *$ \\
\hline & $\mathrm{i} / \mathrm{NB}^{\circ}$ & 38,059 & 6,178 & 26,758 & 4,514 & $<0,0001 *$ \\
\hline & $\mathrm{i} / \mathrm{NB}$ & 9,721 & 2,714 & 4,354 & 1,817 & $<0,0001 *$ \\
\hline & $\mathrm{I} / \mathrm{i}^{\circ}$ & & & & 8,320 & $<0,0001 *$ \\
\hline & & 108,485 & 16,439 & 126,806 & & \\
\hline & \multicolumn{6}{|c|}{ Composantes maxillaires } \\
\hline & $\mathrm{SNA}^{\circ}$ & 85,765 & 4,406 & & 3,473 & $<0,0001 *$ \\
\hline & A-N perp & 9,147 & 4,500 & 80,065 & 2,655 & $<0,0001 *$ \\
\hline & \multicolumn{6}{|c|}{$\begin{array}{c}3,774 \\
\text { Composantes mandibulaires }\end{array}$} \\
\hline & \multirow[t]{2}{*}{$\mathrm{SNB}^{\circ}$} & \multicolumn{3}{|c|}{4,779} & 3,539 & $0,003 *$ \\
\hline & & 80,279 & \multirow{3}{*}{9,839} & \multirow[t]{2}{*}{77,065} & & \\
\hline & \multirow[t]{2}{*}{ Co-Gn } & & & & 10,919 & $0,014^{*}$ \\
\hline & & 100,470 & & \multirow[t]{2}{*}{93,968} & & \\
\hline & Pog-N perp & 7,309 & 6,448 & & 5,048 & $0,015^{*}$ \\
\hline & \multirow[t]{3}{*}{ Pog-NB } & $-0,691$ & 0,913 & 3,694 & 0,922 & $<0,0001 *$ \\
\hline & & \multirow{2}{*}{\multicolumn{4}{|c|}{$\begin{array}{c}1,629 \\
\text { ns maxillomandibulaires }\end{array}$}} & \\
\hline & & & & & & \\
\hline & $\mathrm{ANB}^{\circ}$ & 5,485 & 2,743 & 2,871 & 1,722 & $<0,0001 *$ \\
\hline & AoBo & 1,721 & 3,572 & 2,806 & 2,072 & $0,144 \mathrm{~ns}$ \\
\hline & \multirow[t]{3}{*}{ NA-Pog ${ }^{\circ}$} & & & & 3,962 & $<0,0001 *$ \\
\hline & & 168,250 & 5,546 & 175,387 & & \\
\hline & & \multicolumn{5}{|c|}{ Composantes verticales } \\
\hline & $\mathrm{FMA}^{\circ}$ & 24,265 & 5,462 & 22,887 & 2,756 & $0,211 \mathrm{~ns}$ \\
\hline & $\mathrm{SN} / \mathrm{GoGN}^{\circ}$ & 34,574 & 5,885 & 34,435 & 3,313 & $0,909 \mathrm{~ns}$ \\
\hline & $\mathrm{SN} / \mathrm{Occl}^{\circ}$ & 17,868 & 5,393 & 18,742 & 4,219 & $0,472 \mathrm{~ns}$ \\
\hline
\end{tabular}




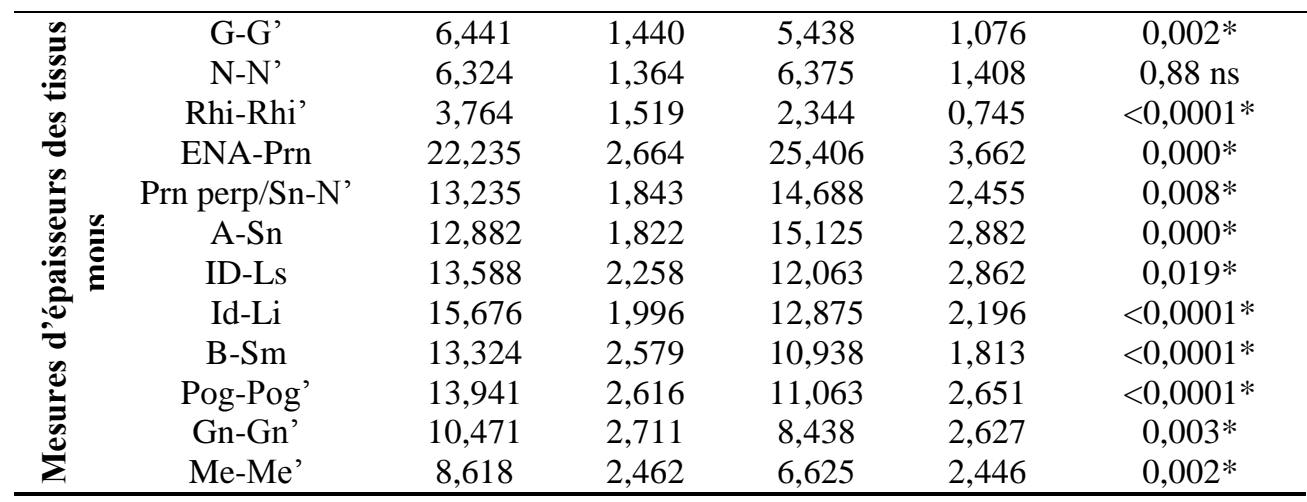

*Valeur de P significative (P<0,005) ns=Valeur de P non significative -Test student-

Les caractéristiques dento-squelettiques et d'épaisseurs des tissus mous du profil cutané des sujets féminins ivoiriennes et libanaises, reflètent la diagnose anthropologique spécifique à chacun de leurs différents groupes ethniques d'origine. Cependant, si le prognathisme facial (SNA $=85,706^{\circ}$, $\mathrm{SNB}=80,176^{\circ}, \mathrm{A}-\mathrm{NPerp}=9,147^{\circ}, \operatorname{Pog}-\mathrm{Nperp}=7,176^{\circ}, \mathrm{I} / \mathrm{i}=108,485^{\circ}, \mathrm{I} / \mathrm{NA}=$ $5,956 \mathrm{~mm}$ et $\mathrm{i} / \mathrm{NB}=9,721^{\circ},\left(\mathrm{I} / \mathrm{NA}=26,412^{\circ}\right.$ et $\mathrm{i} / \mathrm{NB}=38,059^{\circ}, \mathrm{NA}-\mathrm{Pog}=$ $168,25^{\circ}$ ) est bien notable chez les filles ivoiriennes, la divergence faciale semble moins marquée par rapport aux libanaises [FMA $\left.{ }^{\circ}(\mathrm{P}=0,211 \mathrm{~ns})\right]$ contrairement à ce qui a été donné d'observer chez les sujets masculins. La longueur mandibulaire chez les filles ivoiriennes $(\mathrm{Co}-\mathrm{Gn}=100,470 \mathrm{~mm})$ est plus importante. La proximité morphologique relative aux épaisseurs des tissus mous chez les deux groupes de sujets féminins est quasi inexistante, dans la mesure où excepté la racine du nez $\left[\mathrm{N}-\mathrm{N}^{\prime}(\mathrm{P}=0,88 \mathrm{~ns})\right]$ la densité de l'enveloppe peaucière et cutanée est significativement fluctuante dans tous les compartiments du visage. Ainsi, outre les épaisseurs des tissus mous caractéristiques des deux groupes de sujets telles que déjà mentionnées (nez plus proéminent donc plus épais chez les libanaises et lèvres plus épaisses chez les ivoiriennes), le front $\left(\mathrm{G}-\mathrm{G}^{\prime}=6,441 \mathrm{~mm}\right)$ et le menton (Pog-Pog'= 13,941 mm, Gn-Gn'=10,471mm, Me-Me'=8,618mm) des filles ivoiriennes sont plus épais.

\section{Discussion}

La céphalométrie comparative, à travers différentes études précisant la diagnose anthropologique des populations humaines, a permis de révéler l'extrême variabilité morphologique craniofaciale et des épaisseurs des tissus mous du visage.

Cette démarche, d'un intérêt certain pour l'anthropologie médicolégale a orienté ce travail de comparaison des caractéristiques dentosquelettiques et d'épaisseurs des tissus mous des 119 patients adolescents [62 ivoiriens (34 filles et 28 garçons) et 57 libanais (31 filles et 26 garçons)] de 11 à 16 ans, de classe I et normodivergents, à partir de radiographies extraites 
des dossiers médicaux. Ces sujets, d'une biparentalité ivoirienne et libanaise, et dont les angles ANB et FMA correspondent aux valeurs de normalité propres à leurs groupes ethniques d'appartenance, traduisent la réalité de la mixité sociale en Côte d'Ivoire. Il s'agissait donc de deux populations compatibles au regard du genre (sujets masculins et féminins) et de la distribution de l'âge (11 à 16 ans).

Comparés aux leuco-libanais, du point de vue de l'architecture dentosquelettique, les sujets ivoiriens présentent un prognathisme facial se traduisant par une biprotrusion plus marquée des bases alvéolaires et une vestibuloversion des incisives centrales à l'origine du caractère plus obtus de l'angle de convexité faciale. Laquelle convexité faciale révèle en effet une très faible proéminence du menton. Des études antérieures décrivant le profil typologique du complexe craniofacial négro-africain [Kapila, (1987); Faustini et al. (1996); Huang et al. (1997); Utomi (2004)] avaient déjà révélé ces éléments de différentiation spécifiques. Au-delà de la conformation des composantes mandibulaires, maxillaires et dento-alvéolaires traditionnellement évoquées comme déterminants du prognathisme facial, certains auteurs ont développé d'autres argumentaires pour expliquer cette particularité morphologique faciale du sujet négro-africain. Pour ces derniers (Bacon, 1983; Barter, 1995), la longueur du segment antérieur de la base du crâne peut influencer la position antéropostérieure du nasion et donc affecter les valeurs des angles SNA et SNB; ce qui devrait donc être pris en considération lorsque l'on compare deux groupes ethniques différents. Selon Enlow et Hans (1996), la base du crâne est le pont entre le neuro-crâne et le crânium facial à partir duquel la face est construite, par conséquent, les variations au niveau de la base du crâne sont associées à une variation correspondante dans la forme de la face (Kasai et al. 1993). Comme les sujets mélano-africains présentent une base du crâne plus courte, des valeurs plus importantes devraient être attendues pour les angles SNA et SNB (Naidoo, 1997; Bacon, 1983; Barter, 1995). Les données relatives aux angles SNA et SNB des ivoiriens (significativement plus grandes), confortent cet argumentaire, et laissent envisager la possibilité d'un segment antérieur de la base du crâne plus bref chez ces derniers, à cause justement de la position antéropostérieure du nasion. Ce qui, par conséquent retentit sur la valeur de l'angle ANB, dans la mesure où quoique plus grand l'angle SNB ne l'était pas suffisamment pour compenser l'importance de l'angle SNA (Dandajena et Nanda, 2003). Aussi, la plus faible proéminence du menton mélanoivoirien, déjà notée par Freitas et al. (2010) lorsqu'ils comparaient deux fractions de populations négroïdes et caucasoïdes brésiliens, révèle en réalité une cinétique de croissance faciale plus importante dans le sens vertical (FMA plus grand) comme l'indiquaient dans des études précédentes, Freitas et al. (2010), Dandajena et Nanda (2003), Carter et Slattery (1988). 
La comparaison des genres entre les deux populations étudiées, met en relief une proximité morphologique au niveau de la divergence faciale (FMA) chez les deux groupes de sujets féminins, mais surtout, une longueur mandibulaire plus importante chez les filles ivoiriennes, révélant ainsi une croissance mandibulaire plus accrue dans le sens de la longueur chez ces dernières. La croissance en longueur de la mandibule est assujettie à la position du maxillaire, ceci afin de maintenir la permanence de l'occlusion des dents durant la période d'éruption de celles-ci (Petrovic et al. 1979). La longueur effective de la mandibule observée chez les filles ivoiriennes n'est donc que l'une des conséquences notables de la protrusion maxillaire précédemment désignée comme particularisant le sujet mélanoderme.

Le retentissement cutané de cette architecture dento-squelettique prognathe caractérisant les sujets mélano-ivoiriens, est manifeste à travers des lèvres plus protruses, donc, plus épaisses ; justifiant ainsi les fortes épaisseurs des tissus mous péri-buccaux de soutien (base de la lèvre inférieure et au niveau du menton) observées. Lesquelles caractéristiques d'épaisseurs des tissus mous labiaux et péri-labiaux traduisent des caractéristiques négroïdes telles que déjà mentionnées dans la littérature par Djaha et al. (1996) en Côte d'ivoire, Isiekwe et al. (2012) au Nigéria. Aussi, le nez des sujets mélanoivoiriens, platyrhinien, bas et plongeant présente, comparativement aux sujets leuco-libanais, un dos plus épais et une faiblesse générale des épaisseurs des tissus mous péri-nasaux de soutien, en conformité avec les observations de Isiekwe et al. (2015) sur des sujets négroïdes nigérians. Cependant, quoique présentant les caractéristiques générales d'épaisseurs des tissus mous attribuées aux sujets négroïdes, les sujets masculins mélano-ivoiriens sont beaucoup plus proches des sujets masculins libanais au niveau des épaisseurs des tissus mous dans toute la région périphérique du menton. Laquelle proximité morphologique d'épaisseurs des tissus mous est quasi inexistante chez les deux groupes de sujets féminins, dans la mesure où excepté la racine du nez, la densité de l'enveloppe peaucière et cutanée est significativement fluctuante dans tous les compartiments du visage. Ainsi, outre les épaisseurs des tissus mous caractéristiques des deux groupes de sujets (nez plus proéminent donc plus épais chez les libanaises et lèvres plus épaisses chez les ivoiriennes), le front et le menton des filles ivoiriennes présentent des densités de structures molles plus importantes, à peu près à l'image de ce que les données de Cavanagh et al. (2011) ont mis en relief chez un groupe de sujets féminins mélano-sud-africaines.

Les particularités morphologiques de l'architecture dentosquelettique des sujets libanais (angle de convexité facial plus ouvert traduisant un orthognathisme facial, une plus forte proéminence du menton, une plus faible divergence faciale) observées dans cette étude sont conformes aux caractéristiques mises en évidence chez un groupe de leuco-libanais par 
Ayoub et al. (2008). Cette morphométrie dento-squelettique libanaise est également proche des caractéristiques descriptives des populations moyenorientales, telles que mises en relief par Abu-Tayyem et al. (2011) chez un groupe d'adultes émiratis, Bader et al. (2008) relativement aux normes féminines jordaniennes, Behbehani et al. (2006) chez un groupe d'adolescents koweitiens. Concernant les épaisseurs des tissus mous, le nez plus proéminent (donc plus épais) ainsi que son retentissement sur l'épaisseur des tissus mous sub-nasion chez les sujets libanais, apparaissent comme un élément d'anthropologie morphologique particulier aux populations caucasoïdes. Des travaux sur des adultes libanais (El Hayeck et al. 2016), égyptiens (Bagwan et al. 2014) et turcs (Celikoglu et al. 2015), des adolescents Jordaniens (Hamdan ; 2010) confirment cette spécificité anthropologique, tout comme l'ensemble des autres paramètres d'épaisseurs des tissus mous mesurés dans cette étude.

\section{Conclusion}

Cette étude a porté sur des radiographies numériques extraites des dossiers médicaux de 119 patients adolescents [62 ivoiriens (34 filles et 28 garçons) et 57 libanais (31 filles et 26 garçons)] de 11 à 16 ans, classe I squelettique et normodivergents. Les séries de mesures céphalométriques (linéaires et angulaires) réalisées, ont fait l'objet d'analyses statistiques (test de student et de Mann- Whithney, sur séries indépendantes).

Les caractéristiques donto-squelettiques et d'épaisseurs des tissus mous des sujets mélano-ivoiriens et leuco-libanais observés dans cette étude reflètent la diagnose anthropologique de leurs groupes ethniques d'origine. Le prognathisme facial plus marqué, l'acuité de la convexité faciale ainsi que les fortes épaisseurs des tissus mous du tiers facial inférieur (surtout chez les sujets féminins) particularisant traditionnellement les sujets négro-africains, sont observés. Cependant une proximité morphologique des deux groupes ethniques s'observe chez les sujets masculins relativement aux épaisseurs des tissus mous du menton, mais également chez les sujets féminins quant à la divergence faciale. Cette étude, du fait de ce qu'elle a permis de révéler, présente un intérêt en anthropologie médico-légale et révèle l'importance de la céphalométrie radiologique.

\section{References :}

1. Abu-Tayyem, H.M., Alshamsi, A.H., Hafez, S., \& El Din, E.M. (2011). Cephalometric norms for a sample of Emirates adults. Open Journal of Stomatology, 1 (3), 75-83

2. Ayoub, F., Yehia, M., Rizk, A., Al-Tannir, M., Abi- Farah, A., \& Hamadeh, G. (2008). Forensic norms of female and male lebanese adults. Journal of Forensic Odontostomatology, 27 (1), 18-23 
3. Bacon, W., Girardin, P., \& Turlot, J.C. (1983). A comparison of cephalometric norms for the African Bantu and a Caucasoid population. European Journal of Orthodontics, 5 (3), 233-240.

4. Bader, B.A., Vasiliauskas, A., \& Qadri, A.S. (2008). Comparative cephalometric study of Class II division 1 malocclusion between Lithuanian and Jordanian females. Stomatologija, Baltic Dental and Maxillofacial Journal, 10, 44-48

5. Bagwan, A.A., AL-Shennawy, M.I., \& Alskhawy, M.M. (2015). Evaluation of soft tissue parameters for adults with accepted occlusion using Legan and Burstone analysis. Tanta Dental Journal, 12 (1), 1-6

6. Barter, M.A., Evans, W.G., Smit, G.L., \& Becker, P.J. (1995). Cephalometric analysis of a Sotho-Tswana group. The Journal of the Dental Association of South Africa, 50 (11), 539-544.

7. Behbehani, F., Hicks, E.P., Beeman, C., Kluemper, G.T., \& Rayens, M.K. (2006). Racial Variations in Cephalometric Analysis between Whites and Kuwaitis. The Angle Orthodontist, 76 (3), 406-411

8. Carter, N.E., \& Slattery, D.A. (1988). Bimaxillary proclination in patients of Afro-Caribbean origin. British Journal of Orthodontics, 15 (3), 75-84.

9. Cavanagh, D. \& Steyn, M. (2011). Facial reconstruction: Soft tissue thickness values for South African black females. Forensic Science International, 206 (1), 215.e1.

10. Celebi, A.A., Tan, E., Gelgor, I.E., Colak, T., \& Ayyildiz, E (2013). Comparison of Soft Tissue Cephalometric Norms between Turkish and European-American Adults. The ScientificWorld Journal, 2013.

11. Celikoglu, M., Buyuk, S.K., Ekizer, A., Sekerci, A.E., \& Sisman, Y. (2015). Assessment of the soft tissue thickness at the lower anterior face in adult patients with different skeletal vertical patterns using cone-beam computed tomography. Angle Orthodontist, 85 (2), 211217.

12. Cheherre, F. (2011). Apports de l'anthropologie dans l'estimation de l'appartenance à une population (Doctoral dissertation).

13. Dahlberg, G. (1940). Statistical methods for medical and biological students. Allen \& Unwin 
14. Dandajena, T. C., \& Nanda, R. S. (2003). Bialveolar protrusion in a Zimbabwean sample. American journal of orthodontics and dentofacial orthopedics, 123 (2), 133-137.

15. Djaha, K., Bamba, M., Bakayoko-Ly, R., \& Toure, S (1996). Dysharmonie dento-dentaire liée au prognathisme facial du sujet négro-africain. Odonto-Stomatologie Tropicale, 75, 9-12

16. El Hayeck, E., Bassil Nassif, N., \& Bouserhal, J. (2016). Tissus mous : Normes conventionnelles et normes basées sur la verticale vraie dans une population libanaise. International Orthodontics, 14 (3), 311-327

17. Enlow, D.H., \& Hans, M.G. (1996). Essentials of facial growth. WB Saunders Co Philadelphia, 193-199

18. Faustini, M.M., Hale, C., \& Cisneros, G.J. (1997). Mesh diagram analysis: Developping a norm for african americans. Angle orthodontist, 67 (2): 121-128

19. Fitz, C. R. (1981). Radiological evaluation of craniofacial anomalies. Scandinavian journal of plastic and reconstructive surgery, 15 (3), 199-204.

20. Freitas, L.M.A., Freitas, K.M.S., Pinzan, A., Janson, G., \& Freitas, M.R. (2010). A Comparison of Skeletal, Dentoalveolar And Soft Tissue Characteristics In White And Black Brazilian Subjects. Journal of Applied Oral Science, 18 (2), 135-42

21. Gu, M., Mc Grath, C. P.J., Wong, R.W.K., Hägg, U., \& Yang, Y. (2014). Cephalometric norms for the upper airway of 12-year-old Chinese children. Head \& Face Medicine, 10:38

22. Hamdan, A.M. (2010). Soft Tissue Morphology of Jordanian Adolescents. Angle Orthodontist, 80 (1), 80-85

23. Huang, W.J., Taylor, R.W., \& Dasanayake, A.P. (1998). Determining cephalometric norms for caucasians and african Americans in Birmingham. Angle orthodontist, 68 (6), 503-512

24. Isiekwe, G.I., da Costa, O.O., \& Isiekwe M.C. (2012). Lip dimension of an adult Nigerian population with normal occlusion. Journal of Comtemporary Dental Practice, 13 (2), 188-193

25. Isiekwe, G.I., da Costa, O.O., Utomi, I.L., \& Sanu, O.O. (2015). Holdaway's analysis of the nose prominence of an adult Nigerian population. Nigerian Journal of Clinical Practice, 18 (4), 548-553 
26. Kapila, S. (1987). Selected cephalometric angular norms in Kikuyu children. Angle orthodontist, 59 (2), 139-144

27. Kasai, K., Richard, L.C., \& Brown T. (1993). Comparative study of craniofacial morphology in Japanese and Australian aboriginal populations. Human biology, 65, 821-834

28. Meka, M., Sandipamu, T.R., Reddy, R.E., Natta, S., Aduri, R., \& Dande, S.S. (2015). Establishing lateral cephalometric norms for Nalgonda children with mixed dentition. Journal of Orthodontic Research, 3 (2), 134-137

29. Naidoo, L. C., \& Miles, L. P. (1997). An evaluation of the mean cephalometric values for orthognathic surgery for black South African adults. Part 1: Hard tissue. The Journal of the Dental Association of South Africa, 52 (7), 495-502.

30. Petrovic, A., Stutzmann, J.J., \& Gasson, N. (1979). The final length of mandible: Is it genetically determined? L'orthodontie française, 50 , 751-767

31. Purmal, K., Alam, M.K., \& Zam Zam, N.M. (2013). Cephalometric Comparison of Skeletal, Dental, Soft Tissue, Nose and Chin prominence between Malaysian Indian and Malaysian Chinese. International Medical Journal, 20 (3), 335 - 341

32. Purmal, K., Alam, M.K., \& Zam Zam, N.M. (2013). Cephalometric Norms of Malaysian Adult Chinese. International Medical Journal, 20 (1), 87 - 91

33. Utomi, I.L. (2004). A cephalometric study of antero-posterior skeletal jaw relationship in Nigeria Hausa-Fulani children. West African Journal of Medecine, 23 (2),119-22

34. Utsuno, H., Kageyama, T., Uchida, K., Yoshino, M., Miyazawa, H., \& Inoue, K. (2010). Facial soft tissue thickness in Japanese children. Forensic Sciences Internal, 199 (1-3), 109.e1-6.

\section{8-ORAL A}

